\title{
NUMERICAL INVESTIGATIONS OF AERODYNAMIC NOISE DUE TO FLOW PAST A BLUFF BODY
}

\author{
Sultan Ibrahim Alqash \\ PhD Student, ARL-MLS Lab \\ MIE Department, University of Toronto \\ Toronto, Canada \\ sultan.alqash@mail.utoronto.ca
}

\author{
Kamran Behdinan \\ Professor, ARL-MLS Lab \\ MIE Department, University of Toronto \\ Toronto, Canada \\ behdinan@mie.utoronto.ca
}

\begin{abstract}
Landing gears (LG) are primarily designed to support all the loads of an aircraft during landing, taxiing, and taking off. To ease inspection and maintenance, the aerodynamic design is not refined, where many components are exposed to the air flow generating what so-called aeroacoustics noise. To understand the underline physics and investigate both the flow field and its associated acoustic field, a two-dimensional (2D) case of flow past a circular cylinder was simulated using ANSYS Fluent. Two different Reynolds numbers, $R e, 150$ and 90,000 were examined. For low $R e$, two distinct numerical conditions steady and unsteady flow were simulated and compared to examine the effect of the time dependency on the associated acoustic field. For high $R e$, the acoustic field was computed using the built-in Ffowcs William and Hawkings (FW-H) acoustic analogy solver in Fluent. The results show the importance of considering the time variable to extract the corresponding flow data. The far-field noise prediction highly depends on the location of the near-filed data and its associated integral source terms (surface/volume).
\end{abstract}

Keywords-Reynolds number; acoustic analogy; near-field; farfield; pressure fluctuations

\section{INTRODUCTION}

The aircraft noise was recognized as an environmental problem in the early 1950 s, when the turbo-jet powered aircraft was introduced to the markets. Since then, the aircraft noise issue has been dominated by jet noise, known as propulsive noise. In the early 1970s, aircraft noise was successively reduced after the introduction of the first twin cycle bypass turbofan engines [1]. Due to the noise reduction of the propulsive noise that originates from jet-engines, the airframe itself becomes the major contributor to the overall noise. As a result of decades of research and development into noise reduction, by the year 2000 the aircraft noise level had become 20-30 dB quieter compared to the first generation of turbofan powered aircraft. The US and European communities have set a target to minimize the noise level by $50 \%$ near airport areas by 2020 [2]. By 2050, the overall noise level emission of flying aircraft should be further reduced by $65 \%$ [3]. When the aircraft approaches the airport, the engines are almost at low speed which reduces its noise contribution. That yields to put a high portion of the generated noise on the airframe itself. Among the airframe components, the landing gears have been identified as a main source of noise during the approach phase, when they are fully deployed prior to landing for both aircraft types: long range and short range. It has a significant noise level due to its complex structural design that consists of many components with different lengths and sizes.

For a commercial aircraft, the noise generated from the airframe has received much attention in recent years. This has led the Federal Aviation Administration (FAA) to impose strict regulations on the aircraft noise level [4]. All these demands created pressure on industries and academic communities to develop improved techniques for noise reduction and better methods for its prediction. To ease inspection and maintenance, the aerodynamic design is not refined. As a result, many components such as, hydraulic cables, electric wiring, torque links, front and rear braces are exposed to the air flow. Therefore, the flow separation over the LG components constitutes the main noise source mechanism through unsteady wake flow and large-scale vortex instability and deformation [5]. In the literature, there are three distinct approaches to study flow field and associated acoustic field around the LG within certain limitations. Those approaches are experimental, empirical, and numerical. The main difference between flow around landing gears and that around simple geometries, like single or tandem circular cylinders, is the three-dimensionality flow characteristic. For that reason, the experimental studies for the isolated or installed LG system are recommended but too expensive. Thus, the numerical approach becomes an alternative candidate to resolve this issue. Although current computational approaches are infeasible to handle all the turbulence scales that inevitably exist in cases of complex geometry such as a LG, there are some computational fluid dynamic (CFD) approaches that have been utilized to resolve this issue. The most popular methods are Direct Numerical Simulations (DNS), Large-Eddy Simulations (LES), and Reynolds Averaged Navier-Stokes (RANS). Recently, there is a common CFD method known as Detached-Eddy Simulations (DES). The DES approach is a combination of LES and RANS 
solvers, the LES takes care of the large-scale separated flow regions, while RANS handles the modeling of the turbulence boundary layer [6].

Any aeroacoustics problem consists of two fields as its name implies fluid dynamics and acoustics, respectively. It is a two-step process: 1) sound generation based on the study of the aerodynamic flow which generates sound/noise sources and then, 2) sound propagation based on the investigation of the acoustic field spreading in a medium at rest that generated from step 1. The Lighthill's acoustic analogy is considered as the first pioneering work in sound generated aerodynamically and is named after Jims Lighthill [7] in 1952. In 1954, he assumed that the turbulent fluctuations of the stream are equivalently represented by a distribution of quadrupole sources in the same volume of the flow. In 1955, Curle [8] modified the Lighthill's analogy to include the effect of solid boundaries. In 1969, Ffowcs William and Hawkings [9] considered both the influence of the solid surfaces and the turbulence in arbitrary motion and developed the FW-H analogy. The FW-H analogy is considered as the generalized Lighthill's acoustic analogy which makes it more common and applicable for a wide range of applications. Van Mierlo et al. [10] studied the influence of the main landing gear (MLG) inclination angle on the noise level using ANSYS Fluent. However, they did not include the contribution of the quadrupole sources corresponds to the volume integral due to its high computational cost. Thus, the selection of the surface always coincides with the wall of the bluff body. The same approach was used by Long et al. [11] to study the far-field noise of a simplified nose landing gear (NLG). They noticed that the directivity of the noise is a dipole like source. Another method based on CFD and the FW-H equation with penetrable surface has advanced considerably. For instance, Lockard et al. [12] compared the results and noticed that the noise predicted based on solid surface agreed well with the near-field CFD results, and that noise predicted based on the penetrable surface was highly suspected to be corrupted by the pseudo-sound created at the closing FW-H surface in the wake region. Also, Spalart et al. [13] and Sanders et al. [14] argued that by calculating the noise based only on a solid surface would lead to inaccurate results even at a Mach number as low as 0.115 . The results revealed a typical discrepancy of $3 \mathrm{~dB}$ compared to the permeable surface.

In this study, since the LG system is too complex and contains multiple bluff bodies, 2D flow past a circular cylinder with two different Re numbers were simulated. This simplified case will pave the road to understand the underlying physics of both flow structure (near-field) as well as its associated noise propagation (far-field). Different turbulent models were examined according with the flow anticipated behavior (laminar/turbulent). The FW-H solver in Fluent was utilized to study the far-field noise prediction.

\section{ACOUSTIC ANALOGY FORMULATIONS}

\section{A. Lighthill Acoustic Analogy}

Lighthill formulated a wave equation of acoustic analogy where the source derived by comparing the exact equations of motion of a fluid with the equations of sound propagation in a medium at rest as follows [7],

$$
\begin{gathered}
\frac{1}{c_{o}^{2}} \frac{\partial^{2} \rho^{\prime}}{\partial t^{2}}-\nabla^{2} \rho^{\prime}=\frac{\partial^{2} T_{i j}}{\partial x_{i} \partial x_{j}} \\
\mathrm{~T}_{\mathrm{ij}}=\rho v_{\mathrm{i}} \mathrm{v}_{\mathrm{j}}+\mathrm{P}_{\mathrm{ij}}-\mathrm{c}_{\mathrm{o}}^{2} \rho^{\prime} \delta_{\mathrm{ij}} .
\end{gathered}
$$

where $\left(\rho^{\prime}=\rho-\rho_{\mathrm{o}}\right)$ indicates density perturbation, $\mathrm{T}_{\mathrm{ij}}$ is the Lighthill's stress tensor, $\mathrm{P}_{\mathrm{ij}}=\mathrm{p}^{\prime} \delta_{\mathrm{ij}}-\tau_{\mathrm{ij}}$ refers to the compressive stress tensor with $\mathrm{p}^{\prime}$ being the fluctuating pressure and $\tau_{\mathrm{ij}}$ being the total stress tensor equal to $\sigma_{\mathrm{ij}}-\mathrm{p} \delta_{\mathrm{ij}}$ with $\sigma_{\mathrm{ij}}$ refers to the residual stress tensor. Note that $\mathrm{v}_{\mathrm{i}}$ and $\mathrm{v}_{\mathrm{j}}$ indicate the velocity components in $x_{\mathrm{i}}$ and $x_{\mathrm{j}}$ directions, respectively, and $\mathrm{c}_{\mathrm{o}}$ is the speed of sound.

\section{B. Ffowcs Williams and Hawkings Acoustic Analogy}

Curle [8] expanded the Lighthill's analogy considering a rigid surface; after that, Ffowcs Williams and Hawkings further generalized the theory considering a rigid object in arbitrary motion as follows [9],

$$
\begin{gathered}
\left(\frac{\partial^{2}}{\partial \mathrm{t}^{2}}-\mathrm{c}_{\mathrm{o}}^{2} \frac{\partial^{2}}{\partial \mathrm{x}_{\mathrm{i}}^{2}}\right)\left(\mathrm{H}(f) \rho^{\prime}\right)=\frac{\partial^{2}}{\partial \mathrm{x}_{\mathrm{i}} \partial \mathrm{x}_{\mathrm{j}}}\left(\mathrm{T}_{\mathrm{ij}} \mathrm{H}(f)\right)-\frac{\partial}{\partial \mathrm{x}_{\mathrm{i}}}\left(\mathrm{F}_{\mathrm{i}} \delta(f)\right)+\frac{\partial}{\partial \mathrm{t}}(\mathrm{Q} \delta(f)) \\
F_{i}=\left(P_{i j}+\rho v_{i}\left(v_{j}-u_{j}\right)\right) \frac{\partial f}{\partial x_{j}} \\
Q=\left(\rho_{o} u_{i}+\rho\left(v_{i}-u_{i}\right)\right) \frac{\partial f}{\partial x_{i}} .
\end{gathered}
$$

Where $f$ represents the body surface as function of $f(x, t)=$ 0 ; in which $f<0$ and $f>0$ refer to inside and outside of rigid body, respectively. The right-hand side of (3) is the source terms; the first term shown explicitly in (2) physically means unsteadiness inside the fluid expressed in quadruple source as Reynolds stress which is called turbulent-induced term, the second term is dipole source due to local fluctuating stresses exerted by the surface on the fluid and third term is monopole source generated by mass-flux fluctuating around the surface. The last two terms are represented in (4) and (5) which are called loading noise and thickness noise, respectively.

The differential form of FW-H shown in (3) can be written in terms of fluctuating pressure based on the following relationship $\left(p^{\prime}=c_{o}^{2} \rho^{\prime}\right)$ which shows the isentropic relation.

There are three important functions used in the above equations namely Kronecker delta, $\delta_{i j}$, Heaviside, $H(f)$, and the derivative of the Heaviside function $H^{\prime}(f)=\delta(f)$ is the Dirac delta. The turbulent term associated with the quadrupole source is in volume integration. Hence, the decision of including the contribution of the volumes in fluid around rigid body to 
integrate and evaluate the integration kernel is difficult. Thus, it requires a better algorithm/numerical technique to handle the computational cost and memory management. This approach is still under investigation and deserves more developments.

\section{NUMERICAL SET UP}

A two-dimensional case of flow past a circular cylinder was simulated using ANSYS Fluent. First, the flow domain was designed using ANSYS Workbench. The computational domain was extended 5 times and 20 times the cylinder of unity diameter, $D$, upstream and downstream, respectively. The vertical dimension is $5 D$ equally in both Y-directions from the cylinder center. Then, the domain was meshed using an unstructured method of a triangular. However, close to the cylinder wall the structured mesh with rectangular shape elements were utilized to account for the boundary layer (BL) effect. The total number of cells is 22370. After that, the boundary conditions (B.C's) were assigned within corresponding input values. All the numerical modeling process is shown in Fig. 1(a), (b), and (c) starts from the domain, mesh, and B.C's, respectively, including the values of B.C's as shown in Table I.

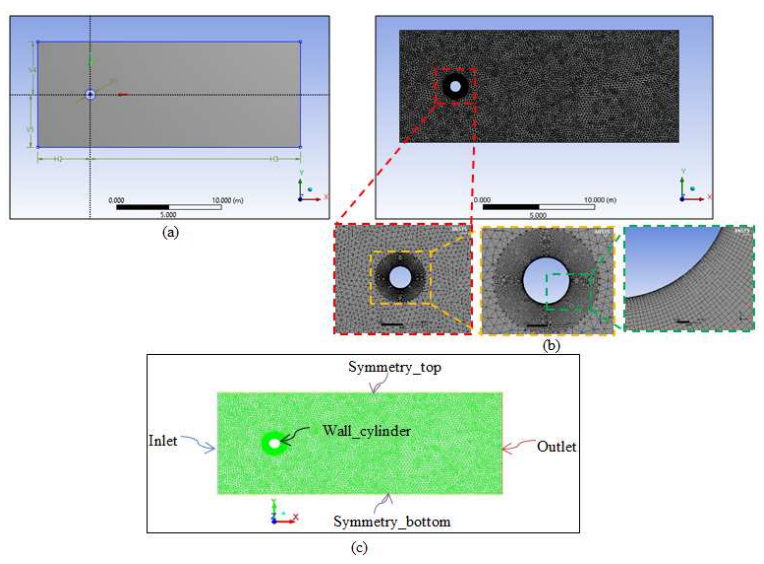

Figure 1. Numerical Modeling: (a) Computational domain, (b) Mesh generation, and (c) Boundary conditions

TABLE I. BOUNDARY CONDITIONS

\begin{tabular}{|c|c|c|}
\hline B.C. & Value & Remarks \\
\hline Inlet & $\mathrm{V}=1[\mathrm{~m} / \mathrm{s}]$ & \multirow{2}{*}{$\begin{array}{l}\text { They are common for } \\
\text { incompressible flows }\end{array}$} \\
\hline Outlet & Gauge Pressure $=0[\mathrm{~Pa}]$ & \\
\hline Wall_cylinder & Stationary wall, $\mathrm{V}_{\text {wall }}=0[\mathrm{~m} / \mathrm{s}]$ & With no-slip condition \\
\hline Symmetry_top & \multirow{2}{*}{ No values needed to be entered } & \multirow{2}{*}{$\begin{array}{l}\text { To ensure that flow } \\
\text { does not form B.L. }\end{array}$} \\
\hline Symmetry_bottom & & \\
\hline
\end{tabular}

Two different Reynolds numbers were examined $R e=150$ and 90,000. In case of low $R e$, two distinct numerical conditions steady and unsteady flow were simulated and compared to examine the effect of the time dependency. For both flow conditions the viscous laminar model was utilized. In case of high $R e$, the acoustic field was computed using the built-in FW-H solver available in Fluent. For this case, the same computational domain is utilized, but the DES with a Spalart-Allmaras (one-equation) model is used to capture the turbulent flow.

\section{RESULTS AND DISCUSSION}

The pressure distribution around the cylinder was computed for both cases and validated with analytical as well as experimental data [16] and showed good agreement as shown in Fig. 2. The analytical pressure coefficient, $C_{p}=1-4 \sin ^{2} \theta$, with respect to different azimuthal angles around the cylinder $\left(0^{\circ} \leq \theta \leq 180^{\circ}\right)$ depicts the symmetrical pattern of the inviscid flow. This symmetry indicates that the integrated surface pressure force in streamwise direction is zero (i.e., no drag force effect). This is an example of the d'Alembert paradox for inviscid flow past an immersed body. However, the experimental data show completely different flow behavior upstream and downstream the bluff body. It strongly depends on the Reynolds numbers as shown in Fig. 2 below, where both the numerical results of laminar and turbulent successfully reproduce the flow behavior upstream and downstream. For the laminar, the separation occurs at around $\theta=87^{\circ}$ which creates a broad wake and very low pressure within large drag coefficient. In contrast, the turbulent flow has a boundary layer that is more resistant which delays the separation point to occur at about $\theta=120^{\circ}$ and then a smaller wake and higher pressure within about $75 \%$ less drag coefficient is resulted.

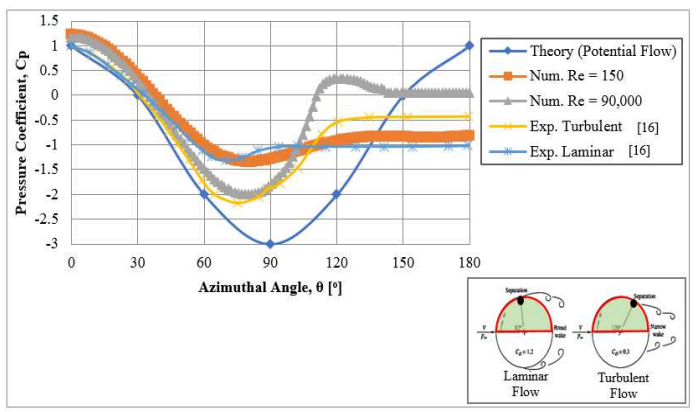

Figure 2. Pressure coefficient profile around the upper surface

The purpose of steady-state simulation is to investigate the mean flow patterns around the bluff-body. The results showed that only the mean velocity and pressure can be captured as shown in Fig. 3(a) and (b), respectively. This is evidence that the acoustic field cannot be extracted, but the results could be utilized as an initial value for simulating unsteady case. On the other hand, the unsteady flow simulation includes the transient behavior which then clearly depicts the flow motion as shown in Fig. 3(c), (d), (e) and (f). The flow instability downstream known as von Karman Street was successfully captured.

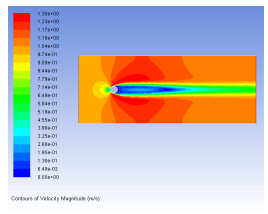

(a)

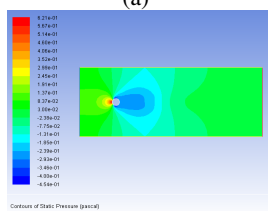

(b)

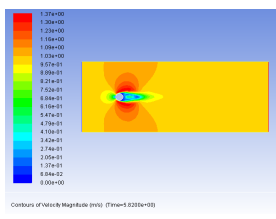

(c)

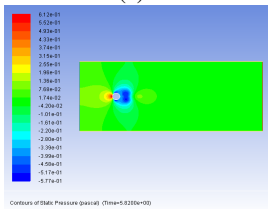

(d)

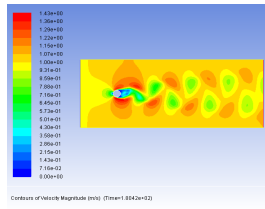

(e)

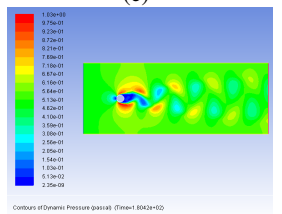

(f)
Figure 3. Near-field results of flow around circular cylinder with $R e=150$ 
Because the flow depends on time, it is important to calculate the time step. For example, in the region behind the circular cylinder a vortex shedding will be created. From the experiments [15], the Strouhal number, $\mathrm{St}=f D / \mathrm{v}$ is 0.172 , then the period, $\mathrm{T}=1 / f$ is found to equal 5.8139 seconds if the frequency, $f$ is calculated. By assuming the number of time steps is $\mathrm{n}=20$, then $\Delta \mathrm{T}=\mathrm{T} / \mathrm{n}=0.291$ seconds, so then, the first vortex shedding can be captured. Note that the lift coefficient, $\mathrm{C}_{\mathrm{L}}$, has a sinusoidal pattern which is an indication of the sustained vortex shedding that takes place in the wake region as shown in Fig. 4.

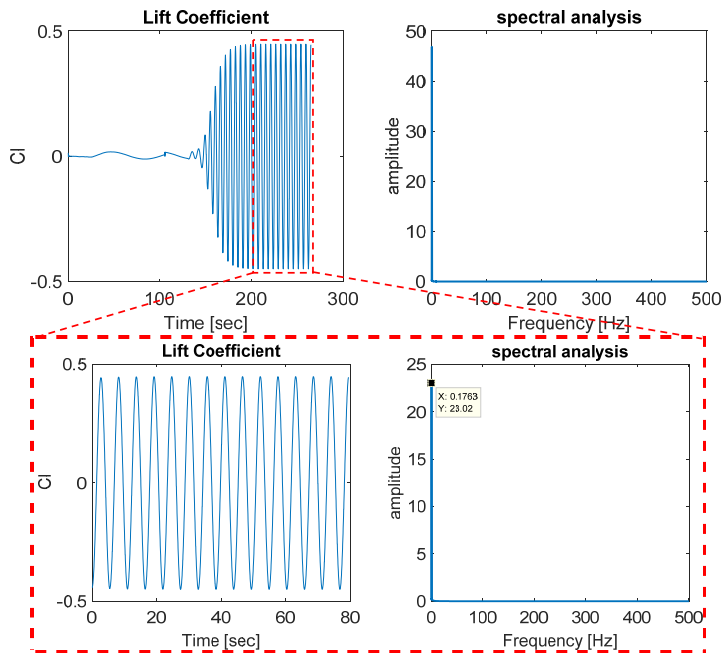

Figure 4. Lift Coefficient time history and frequency content for $R e=150$

The associated acoustic pressure fluctuations for $R e=150$ were collected at two receivers, receiver 1 and receiver 2 located at $90^{\circ}$ above the circular cylinder within $0.665 \mathrm{~m}$ and $2.432 \mathrm{~m}$, respectively. Fig. 5 shows that the far-field pressure fluctuations at both locations preserved the periodicity pattern. That because the near-field flow pressure oscillated with sinusoidal type of behavior when the flow instability occurs.

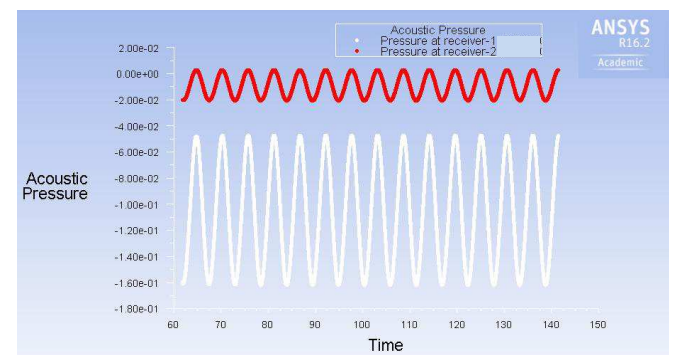

Figure 5. Acoustic pressure fluctuations at two different locations

From Fluent the drag force, $\mathrm{F}_{\mathrm{d}}$, is found to equal $0.696 \mathrm{~N}$, then the drag coefficient, $\mathrm{C}_{\mathrm{d}}$, was calculated using this relation: $\mathrm{C}_{\mathrm{d}}=2 \mathrm{~F}_{\mathrm{d}} / \rho \mathrm{V}^{2} A$, where $A$ is the projected area normal to the flow direction (i.e. for a $2 \mathrm{D}$ cylinder is equal to the diameter), and it turned out to be equal to 1.392 which differs by about $7.2 \%$ from the experimental value [15].

For $R e=90,000$, the near-field data that corresponds to the far-field acoustic prediction is captured as seen in Fig. 6. The wall of the circular cylinder is selected to extract all the relevant acoustic source date such as velocity and pressure. In case of $2 \mathrm{D}$, the source correlation length is assigned to be about $5 D$ since the Fluent assumes that the sound sources are perfectly correlated over the specified correlation length and zero outside. The acoustic pressure signals at the two receiver locations were detected and shown in Fig. 7(a). Also, the spectral of the receiver signals are plotted using the Fast Fourier Transform (FFT). Then, the sound pressure level (dB) versus the frequency $(\mathrm{Hz})$ is calculated as shown in Fig. 7(b) and (c). Due to the turbulence behavior, different size of eddies generated, but the small-scale eddies are not captured within this number of cells. The results prove that the noise generated is a broadband as detected by receivers 1 and 2 . In this case, the pressure fluctuations are no more preserving the periodicity and revealed asymmetric behavior. This indicates that a higher frequency creates a more rapid variation in air pressure and results in a higher pitch.

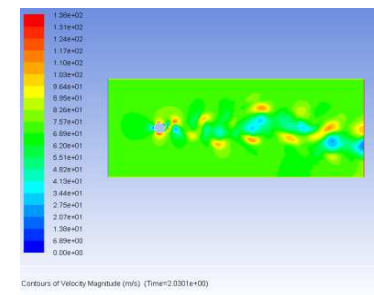

(a)

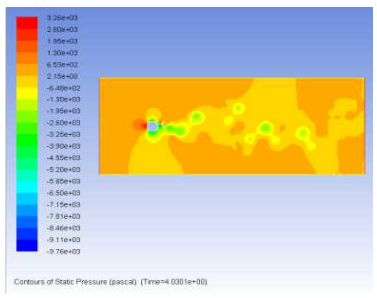

(b)
Figure 6. Numerical results of flow around circular cylinder with $R e=90,000$

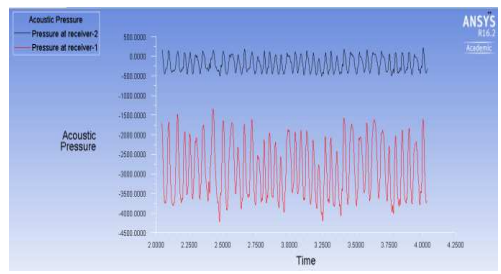

(a)

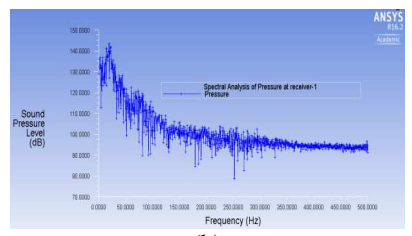

(b)

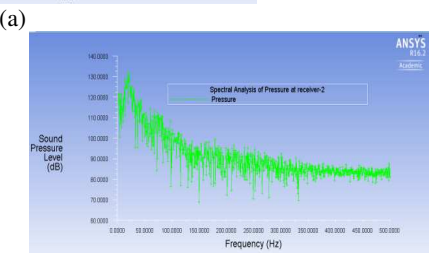

(c)
Figure 7. Acoustic results of flow around circular cylinder with $R e=90,000$

\section{CONCLUSION}

In order to understand the physics of both flow and acoustic fields of landing gear systems, 2D flow past a bluff body (circular cylinder) was simulated using ANSYS Fluent. Two distinct $R e$ numbers 150 and 90,000 were examined. The time dependency consideration affects the extraction of near-field flow data as well as the far-field noise prediction. The FW-H solver in Fluent does not include the contribution of the quadrupole sources (volume integration). Thus, type and location of the surface data are crucial in capturing the far-field noise level. Further investigations are needed to adjust for all source terms. An efficient algorithm/numerical technique in terms of computational cost and memory management are highly required to tackle such complex case like a LG. 


\section{ACKNOWLEDGMENT}

The author is grateful to Prof. Behdinan for supporting and supervising this research. The computing facility provided by his ARL-MLS lab at University of Toronto is gratefully acknowledged. I acknowledge Saudi Arabia government (Taibah University) for granting a scholarship.

\section{REFERENCES}

[1] Morgan, H. G. \& Hardin, J. C. 1975, "Airframe Noise: The Next Aircraft Noise Barrier", Journal of Aircraft, 12 (7), pp. 622-624.

[2] Argüelles, P., Bischoff, M., Busquin, P., Droste, B.A.C., Evans, S.R., Kröll, W., Lagardere, J.L., Lina, A., Lumsden, J., Ranque, D. and Rasmussen, S. 2001, "European Aeronautics: A vision for 2020", Advisory Council for Aeronautics Research in Europe, Report.

[3] Flightpath, A.C.A.R.E., 2011, "2050-Europe's Vision for Aviation", Advisory Council for Aeronautics Research in Europe.

[4] FAA 2009 Airport noise and access restrictions, In FAA Airport Compliance Manual, 5190.6B.

[5] Lazos, B. 2002, "Surface topology on the wheels of a generic four-wheel landing gear", AIAA journal, 40(12), pp. 2402-2411.

[6] Hedges, L. S., Travin, A. K. \& Spalart, P. R. 2002, "Detached-Eddy Simulations Over a Simplified Landing Gear", Journal of Fluids Engineering, 124 (2), pp. 413-423.

[7] Lighthill, M.J. 1952, "On sound generated aerodynamically: I. General theory", In Proceedings of the Royal Society of London A:
Mathematical, Physical and Engineering Sciences, 211(1107), pp. 564587.

[8] Curle, N. 1955, "The influence of solid boundaries upon aerodynamic sound", In Proceedings of the Royal Society of London A: Mathematical, Physical and Engineering Sciences, 231(1187), pp. 505-514.

[9] Williams, J.F. and Hawkings, D.L. 1969, "Sound generation by turbulence and surfaces in arbitrary motion", Philosophical Transactions of the Royal Society of London A: Mathematical, Physical and Engineering Sciences, 264(1151), pp. 321-342.

[10] Van Mierlo, K.J., Takeda, K. and Peers, E. 2010, "Computational analysis of the effect of bogie inclination angle on landing gear noise", In 16th AIAA/CEAS Aeroacoustics Conference, AIAA Paper, pp. 7-9.

[11] Long, S.L., Nie, H. and Xu, X. 2012, "Aeroacoustic study on a simplified nose landing gear", In Applied Mechanics and Materials, pp. 18-23.

[12] ] Lockard, D.P., Khorrami, M.R. and Li, F. 2004, "High resolution calculation of a simplified landing gear", AIAA paper, pp. 2887.

[13] Spalart, P.R., Shur, M.L., Strelets, M.K. and Travin, A.K. 2011, "Initial noise predictions for rudimentary landing gear", Journal of Sound and Vibration, 330(17), pp. 4180-4195.

[14] Sanders, L., Manoha, E., Ben Khelil, S. and Francois, C. 2011, "LAGOON: CFD/CAA coupling for landing gear noise and comparison with experimental database", American Institute of Aeronautics and Astronautics.

[15] Shames, I. H. 1982, Mechanics of Fluid, $3^{\text {rd }}$ Ed., Ch.13: pp. 669-675, McGraw-Hill, New York.

[16] Nakayama, Y. and Boucher, R. 1998. Introduction to fluid mechanics, Butterworth-Heinemann. 